\title{
Elastic anisotropy estimation from laboratory measurements of velocity and polarization of quasi-P-waves using laser interferometry
}

\author{
Maxim Lebedev ${ }^{1}$, Andrej Bóna ${ }^{1}$, Roman Pevzner ${ }^{1}$, and Boris Gurevich ${ }^{2}$
}

\begin{abstract}
A new method for conducting laboratory measurements of the velocities and polarizations of compressional and shear waves in rock samples uses a laser Doppler interferometer (LDI). LDI can measure the particle velocity of a small $(0.03$ $\mathrm{mm}^{2}$ ) element of the surface of the sample along the direction of the laser beam. By measuring the particle velocity of the same surface element in three linearly independent directions and then transforming those velocities to Cartesian coordinates, three orthogonal components of the particle-velocity vector are obtained. Thus, LDI can be used as a localized three-component (3C) receiver of ultrasonic waves, and, together with a piezoelectric transducer as a source, it can simulate a $3 \mathrm{C}$ seismic experiment in the laboratory. Performing such $3 \mathrm{C}$ measure-
\end{abstract}

ments at various locations on the surface of the sample produces a 3C seismogram, which can be used to separate the P-wave and two $\mathrm{S}$-waves and to find the polarizations and traveltimes of those waves. Then, the elasticity tensor of the medium can be obtained by minimizing the misfit between measured and predicted polarizations and traveltimes. Computation of the polarizations and traveltimes of body waves inside a sample with a given elasticity tensor is based on the Christoffel equation. The predicted polarizations on the surface then are obtained using the anisotropic Zoeppritz equations. The type of velocity measured (phase or group velocity) depends on the acquisition geometry and the material properties. This is taken into account in the inversion procedure. A "walkaway" laboratory experiment demonstrates the high accuracy of this method.

\section{INTRODUCTION}

An estimation of the elastic anisotropy of rocks from laboratory measurements is important for calibration of seismic and acoustic studies of subsurface rocks. Traditionally, the anisotropy of rock samples is determined by measuring wave velocities using the "time-of-flight" method. The compressional- and shearwave velocities of the samples are measured in various directions; from those measurements the density-scaled stiffness tensor is estimated. The wave velocities are determined from the traveltimes of waves generated and recorded by ultrasonic piezoelectric transducers (e.g., Pros and Babuska, 1967; Jech, 1991; Rasolofosaon and Zinszner, 2002). Despite the versatility of piezoelectric transducers as sources and receivers in ultrasonics, however, such experiments have at least two problems: (1) uncertainty in determination of the time of shear-wave arrivals, and (2) uncertainty regarding whether phase or group velocity is measured. In partic- ular, if the size of the wave source is small compared with the dimensions of the sample, the measured velocity is the group velocity. If the size of the source is comparable to the size of the sample, the velocity of a wave propagating in the direction perpendicular to the surface of the source is the phase velocity (also called the wavefront velocity). In intermediate situations, which are typical for ultrasonic experiments, unambiguous interpretation of velocity measurements is difficult (Dellinger and Vernik, 1994; Vestrum, 1994; Siggins and Dewhurst, 2007).

In seismic field measurements, the size of the receivers (geophones, hydrophones) is much smaller than the wavelength of the measured waves. This simplifies estimation of the polarization and, thus, determination of the wave type. Use of wave polarizations as well as velocities provides a robust method for estimating anisotropy in situ (Dewangan and Grechka, 2003). The use of a similar approach in the laboratory requires receivers that are small relative to the wavelength. In this paper,

Manuscript received by the Editor 12 August 2010; revised manuscript received 4 February 2011; published online 23 May 2011.

${ }^{1}$ Curtin University, Department of Exploration Geophysics, Perth, Australia. E-mail: m.lebedev@curtin.edu.au; a.bóna@curtin.edu.au; r.pevzner @ curtin.edu.au.

${ }^{2}$ Curtin University, Department of Exploration Geophysics, Perth, Australia and CSIRO Earth Science and Resource Engineering, ARRC, Perth, Australia. E-mail: gurevich@curtin.edu.au.

(C) 2011 Society of Exploration Geophysicists. All rights reserved. 
we propose a method for measuring anisotropy in laboratory experiments using a laser interferometer.

Laser interferometery has been used for decades to register acoustic wave arrivals in the laser ultrasonics techniques. Those techniques use a pulse laser as a source of elastic waves and a laser interferometer as a receiver (Dainty, 1975; Ennos, 1978; Monchalin, 1986; Monchalin et al., 1989; Scruby and Drain, 1990; Jacquot and Fournier, 2000). Such a technique has been applied to measurements of the elastic properties of isotropic materials (Aussel and Monchalin 1989, Pouet and Rasolofosaon, 1993) and anisotropic materials (Guilbaud and Audoin, 1999, Ogi et al., 2003). In particular, Pouet and Rasolofosaon (1990), Martin et al. (1994), and Rasolofosaon et al. (1994) use laser interferometry to measure polarizations of quasi-S-waves.

Nishizawa et al. (1997) proposes a method of shear-wave detection in the laboratory using laser Doppler interferometry (LDI). That method is based on measurement of the wave-induced movement of a small area of the surface of the sample. To separate $\mathrm{P}$ - and S-waves, those authors performed measurements in two directions and then found projections of the displacement onto directions perpendicular to and parallel to the surface. Bayón and Rasolofosaon (1996) demonstrated that laser speckle interferometry can be suitable for measuring the displacement in normal and tangential directions. They also discuss the fidelity of particle-displacement measurements induced by ultrasonic waves. Fukushima et al. (2003) used LDI to investigate the polarization of the shear waves in rock samples. The main advantages of using LDI instead of piezoelectric transducers are that (1) the area of the measurement is much smaller than the wavelength and (2) the full particle-velocity vector can be recorded.

The objective of this paper is to enhance the estimation of anisotropy using laser interferometry, by including measurements of the ultrasonic wave polarizations. The aim of using polarizations is twofold: first, they aid in distinguishing between the measured waves, and second, they are considered alongside wave velocities in the inversion process. This extra information helps one to constrain the estimation of the anisotropy better than the other methods that rely on traveltime only.

\section{THEORETICAL BACKGROUND}

The propagation of elastic waves within a rock sample is determined by the stiffness tensor and the density of the material. The polarizations of these waves, as well as their phase (wavefront) velocities, are related by the stiffness tensor through the Christoffel equations (e.g., Červený, 2001):

$$
c_{i j k \ell} p_{j} A_{k} p_{\ell}=\rho A_{i}
$$

where $\mathbf{c}$ is the stiffness tensor, $\mathbf{p}$ is the phase slowness, $\mathbf{A}$ is the polarization, and $\rho$ is the density. The direction of the phase velocity $\mathbf{v}$ is always perpendicular to the wavefronts and is the wave speed in this direction. The phase slowness has the same direction as does the phase velocity and has the magnitude of the reciprocal of the phase velocity. The Christoffel equations can be written as an eigenvalue problem for the Christoffel matrix along direction $\mathbf{n}, \boldsymbol{\Gamma}(\mathbf{n})_{i k}=c_{i j k \ell} n_{j} n_{\ell}$, where a polarization $\mathbf{A}$ is the eigenvector and $\rho v^{2}$ is the eigenvalue ( $v$ is the magnitude of the phase velocity). The matrix can have three different eigenvalues corresponding to the three body waves propagating in an elastic material. Because of the symmetry of the Christof- fel matrix, the polarizations corresponding to the three waves are mutually orthogonal.

We note that in general the group (ray) and phase velocities are different, and they are related by the expression

$$
V_{i}=p_{i} v^{2}+p^{2} v \frac{\partial v}{\partial p_{i}},
$$

where $\mathbf{V}$ is the group velocity, $v$ is the magnitude of the phase velocity, and $\mathbf{p}$ is the phase slowness. The group velocities and the polarizations are related by the expression (e.g., Červený, 2001; Bóna et al., 2008)

$$
\boldsymbol{\Gamma}\left(\boldsymbol{\Gamma}^{-1}(\mathbf{A}) \cdot \mathbf{V}\right) \cdot \mathbf{A}=\mathbf{A} .
$$

A thorough discussion of wave propagation in anisotropic media can be found in, for example, Helbig (1994).

Particle displacement on an interface between two materials is given by a superposition of the displacements of the incoming, reflected, and transmitted waves. These displacements are functions of the elastic properties of the two materials and of the direction of the incoming wavefront. The directions of the reflected and transmitted waves can be found by considering Snell's law. The polarizations of the waves are the eigenvectors of the Christoffel matrix in the direction of the wave propagation. The amplitudes of the waves can be found by solving the anisotropic Zoeppritz equations. Combining all this information will produce the displacement direction of a point on the interface.

\section{EXPERIMENT}

\section{Sample and experimental setup}

To measure the wave polarizations and velocities for use in estimating anisotropy, we constructed the experimental setup shown in Figure 1. The apparatus uses a laser Doppler interferometer to find the displacement of a point on the surface of a sample in a given direction. We used a Vibrometer OFV-5000 Modular Vibrometer Controller with Vibrometer Sensor Head OFV-503 (Politec Ltd.). This device can measure velocity and displacement simultaneously in wide frequency range (from $1 \mathrm{~Hz}$ to $2.5 \mathrm{MHz}$ ).

To demonstrate the possibility of measuring polarization, we used an experimental geometry suggested by Nishizawa et al. (1997): We performed a laboratory equivalent of a geophysical "walkaway" survey. Figure 2a shows the spatial arrangement of the experiment. We chose to perform the experiment on paper-reinforced phenolic material, because this synthetic material has a strong anisotropy. The $\mathrm{P}$-wave velocities at an ultrasonic frequency of $1 \mathrm{MHz}$ parallel and perpendicular to the layering plane are $3531 \pm 40 \mathrm{~m} / \mathrm{s}$ and $2887 \pm 30 \mathrm{~m} / \mathrm{s}$, respectively. From the optical images of the surfaces of the sample (shown in the Figure $2 b$ ), it is clear that the distance between layers is approximately $0.2 \mathrm{~mm}$. For this material to behave as if it were effectively homogeneous, following Aki and Richards (2002), the distance between the layers should be smaller than the wavelength divided by $2 \pi$. In our case this condition clearly is satisfied, because the wavelength of the ultrasonic S-wave is $2.6 \mathrm{~mm}$ and that of the $\mathrm{P}$-wave is $3.5 \mathrm{~mm}$, at a frequency of $1 \mathrm{MHz}$. Therefore, for the purpose of our experiments the sample can be considered to be homogeneous.

For the wave source, we glued a 14-mm-diameter S-wave transducer by epoxy directly to the bottom side of the sample, with the polarization of the source oriented at $45^{\circ}$ with respect 
to the $x$ - and $y$-coordinate axes. Such an S-wave transducer generates both $\mathrm{SH}$ - and SV-waves, as well as a P-wave. A strip of reflective tape (manufactured by $3 \mathrm{M}$ ) was glued on the surface of the sample, as shown in Figure 2a. This tape is made from 50 - $\mu$ m-diameter glass microbeads; each glass bead reflects light backward. Reflective tape works ideally for the laser-beam incident angles to as great as $80^{\circ}$ (from the direction normal to the surface). We chose the incident angles of LDI to be $55^{\circ}$ from the normal (z-axis) to the surface of the sample. The measurements were made along a single line with $10-\mathrm{mm}$ spacing. The surface of the material was parallel to the bedding plane. Experimental records of the particle displacement and its velocity in one direction are shown in Figure 3. We see that recorded signals are of very high quality; in our experiments, the signal-tonoise ratio can vary from 10 to 100 .

The spatial components of the particle displacement and velocity on the surface can be measured at different points in at least three

a)

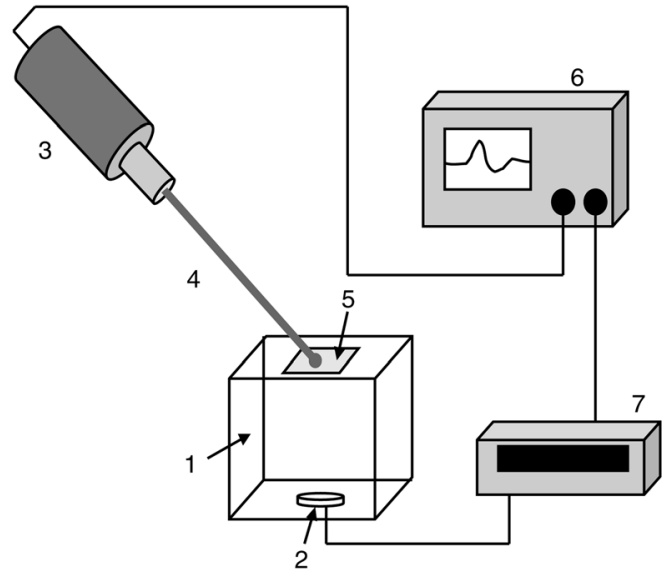

b)

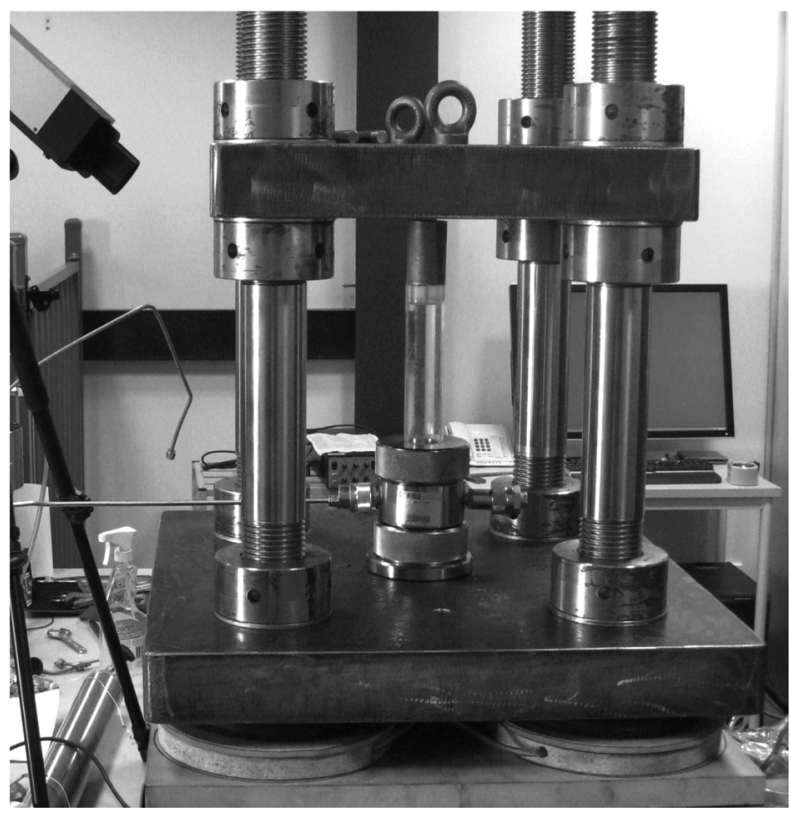

Figure 1. (a) Experimental setup, and (b) experimental rig. 1: sample, 2: source of elastic waves, 3: laser Doppler interferometer, 4: laser beams (emitted and backscattered), 5: reflective film, 6: acquisition system device, and 7: pulse generator. directions (Figure 4). These directions should be chosen so that they span the three-dimensional space. By measuring at least three linearly independent components of the particle velocity and/or the displacement at a particular point, the polarization (direction of particle motion versus time) can be determined. Mathematically, this corresponds to finding a vector from its projections onto three independent vectors. As a result of these measurements, the arrival times of $\mathrm{P}$ - and S-waves, as well as their polarization (direction of particle motion), can be determined.

\section{Measurements}

After measuring the particle velocities in the three linearly independent directions (axes 1, 2, and 3 in Figure 4), we plotted

a)

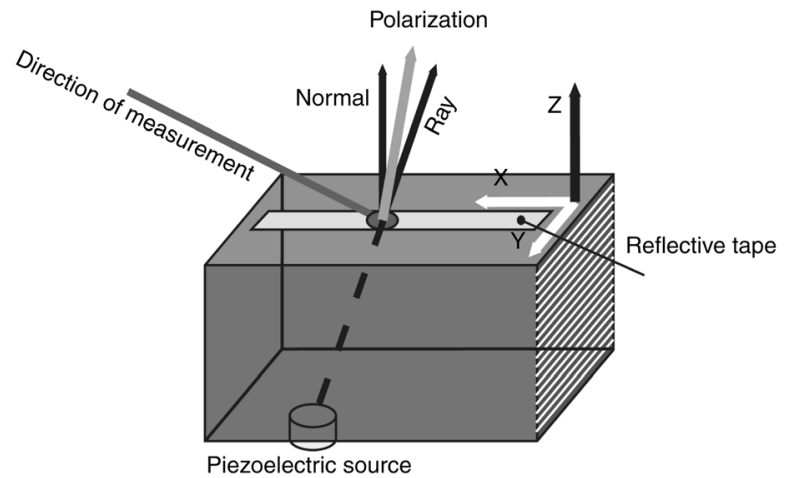

b)
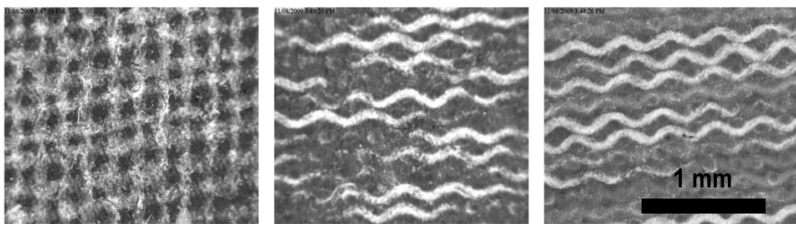

Figure 2. (a) Walkaway experiment setup using a paper-reinforced phenolic sample, and (b) optical images of the surface of the sample. Note that the distance between layers $(0.2 \mathrm{~mm})$ is much smaller then the ultrasonic wavelength $(2 \mathrm{~mm})$, so the media can be considered to be homogeneous.

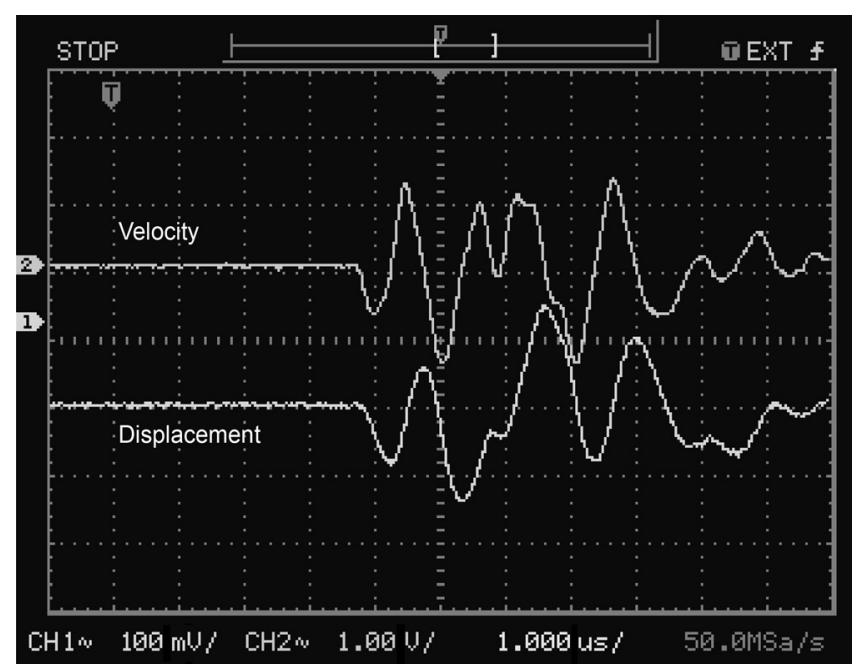

Figure 3. Typical oscilloscope traces directly recorded from LDI. Note the excellent signal-to-noise ratio. 
in Figure 5 the velocities of the surface particle motion versus time, in a Cartesian coordinate system $x, y$, and $z$ (with the $z$ axis perpendicular to the surface, as shown in Figure 3). The size of the sample has been selected so that the registered waveforms do not contain reflected waves from the walls of the sample; the traveltime of the S-waves is shorter than the traveltime of the reflected P-waves.

In a special series of experiments, we investigated repeatability and reliability of registration of the surface velocity. In particular,

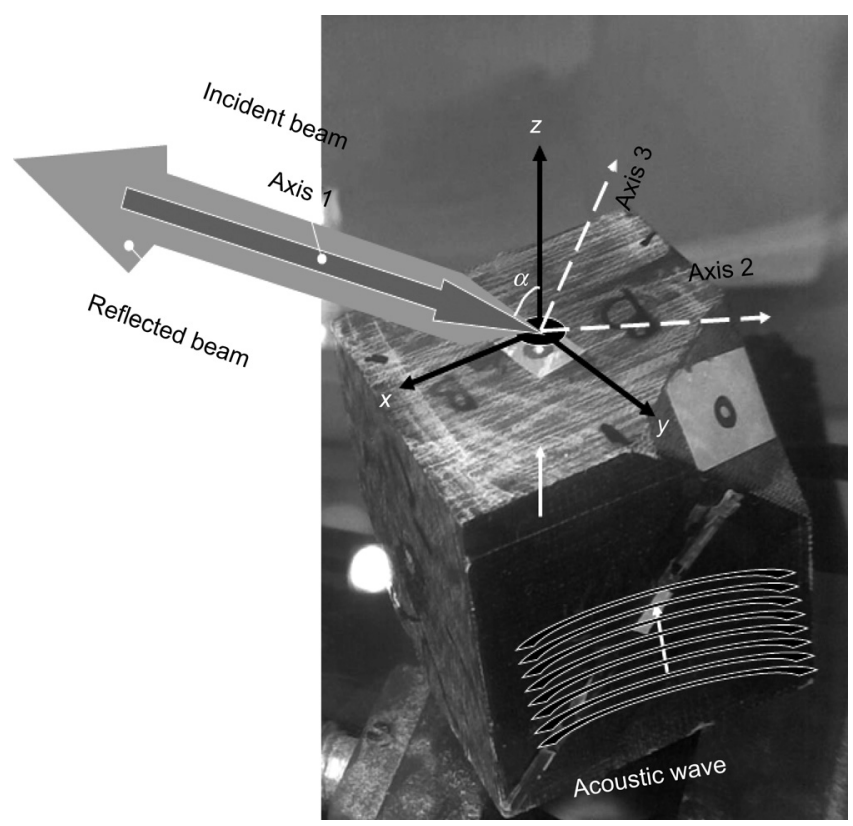

Figure 4. Schematic of the three-component displacement measurement. An image of the sample is embedded in the picture.
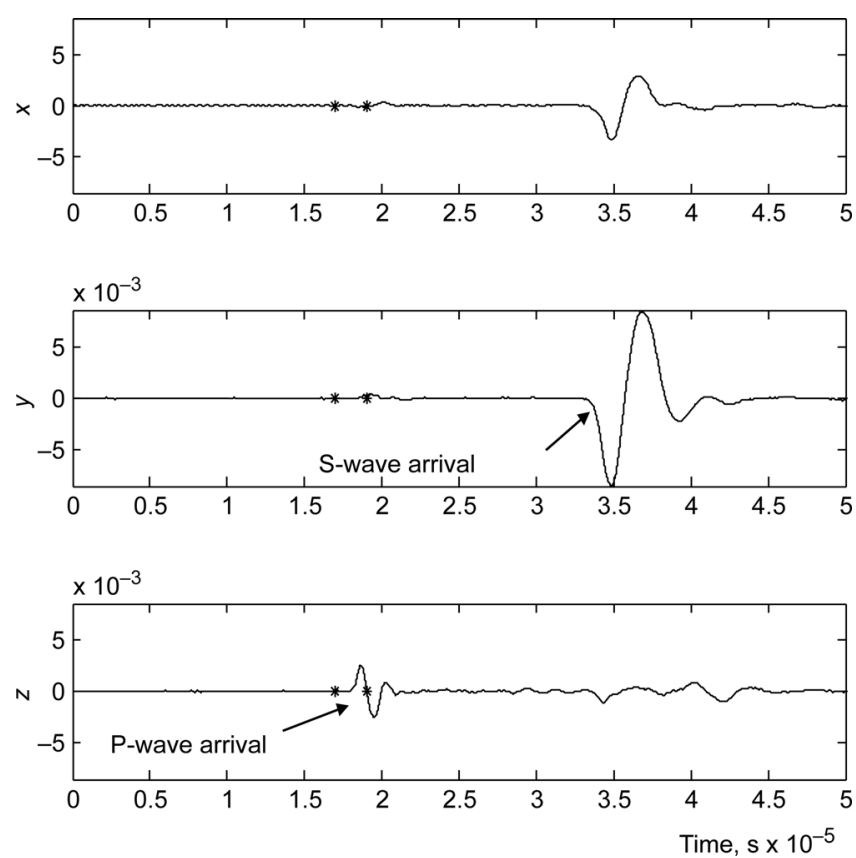

Figure 5. Velocities of the particle motion on the surfaces (arbitrary units) at directions $x, y$, and $z$ (shown in Figure 2a), versus time. we found that misalignment of the laser focus within $0.5 \mathrm{~mm}$ has no influence on the final result. Such misalignment, even for a wave incident angle (ray angle) of $45^{\circ}$, results in an error of wave arrival of $100 \mathrm{~ns}$, or $0.5 \%$, and the uncertainty in estimation of the polarization is less than $1^{\circ}$. The displacements as functions of time can be found by integration of the velocities; an example of the particle trajectory (displacements) for the P-wave is shown in Figure 6. In this example, from Figure $6 \mathrm{a}$ and $6 \mathrm{~b}$ it is clear that the trajectory of the particle motion in the $y-z$ plane is normal to the surface of the sample, whereas in the $x$-z plane it deviates approximately $7^{\circ}$ from the normal. Separation of shear waves with vertical (SV) polarization from those with horizontal $(\mathrm{SH})$ polarization is demonstrated in Figure 7.

Results of the walkaway polarization measurements are shown in Figure 8. The offset indicated in the graph is measured from the edge of the source in order to eliminate doubt about
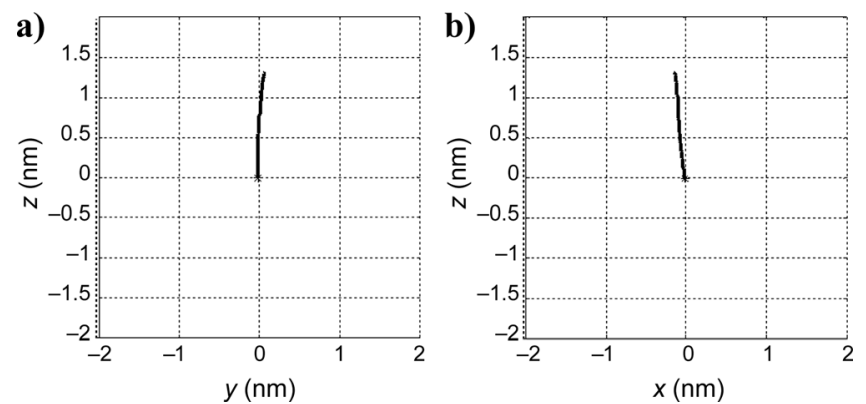

Figure 6. Trajectory of particles at the time interval $11 \sim 14.5 \mu \mathrm{s}$ (between the stars in Figure 5): (a) in the $z-y$ plane, and (b) in the $x-z$ plane.

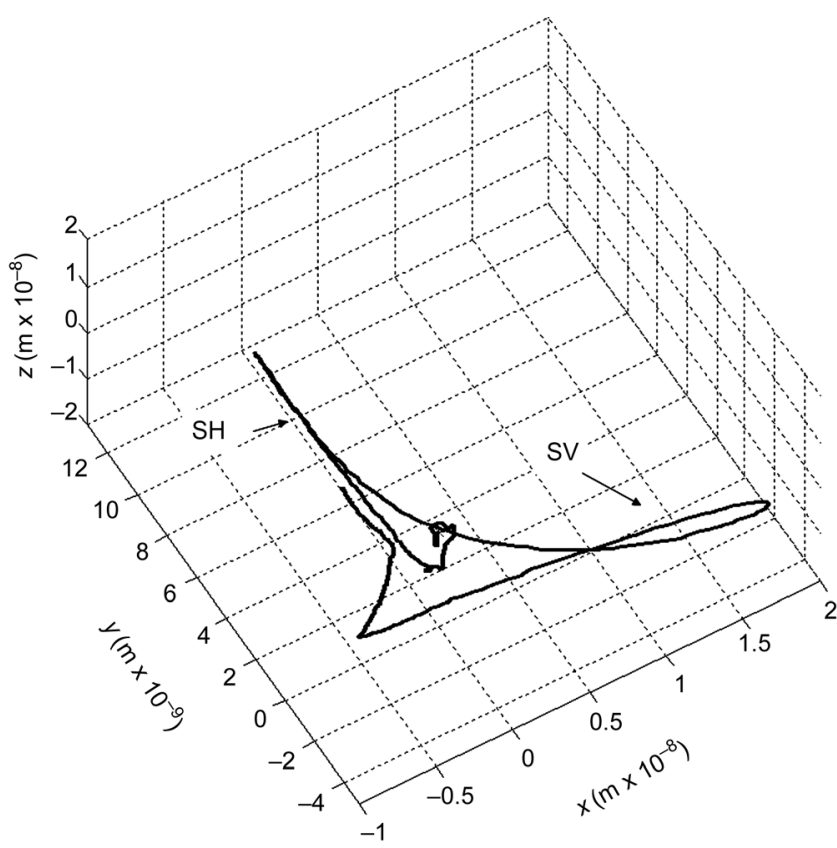

Figure 7. A hodogram showing the splitting of SH- and SVwaves registered by laser Doppler interferometry. The initial position is $(0,0,0)$. The source: $\mathrm{S}$-wave transducer is tilted at $45^{\circ}$ with respect to the axis of symmetry, to generate both SV- and $\mathrm{SH}$-waves. 
the type of the measured wave. In particular, the phase and group velocities are equal along the symmetry axis, whereas they differ for increasing angles from the symmetry axis. Hence, as discussed by Vestrum (1994), the velocities measured away from the edge of the source are the group velocities.

\section{ESTIMATING ELASTIC STIFFNESS COEFFICIENTS}

To show the applicability of laser-interferometry polarization measurements for estimating elasticity, we compare the measured polarizations with polarizations obtained by analytic forward modeling. The modeling is based on measurements of the $\mathrm{P}$-wave velocity and the two S-wave velocities in different directions on the sample. These velocities are converted to the stiffness tensor that is used to model polarizations on the free surface using the Zoeppritz equations.

From the measured polarizations and velocities of the (quasi-) compressional and (quasi-) shear waves, we can find the density-scaled stiffness tensor of the sample. The inversion algorithm is based on numerical minimization of the following misfit function:

$F(a)=\sum_{i=1}^{N}\left\|\mathbf{A}\left(\mathbf{a}, n_{i}\right)-\mathbf{A}_{\text {obs }}\left(n_{i}\right)\right\|^{2}+\alpha\left|v\left(\mathbf{a}, n_{i}\right)-v_{\text {obs }}\left(n_{i}\right)\right|^{2}$,

where $\mathbf{a}$ is a density-weighted stiffness tensor, $N$ is the number of measurements, $n_{i}$ is the measurement direction (source-receiver direction), $\mathbf{A}$ is computed polarization from $a$ in direction $n_{i}, \mathbf{A}_{\text {obs }}$ is observed polarization in direction $n_{i}, v$ is computed ray or phase velocity depending on $n_{i}, v_{\mathrm{obs}}$ is observed velocity, and $\alpha$ is a scaling factor to account for different errors and units of polarization and velocity measurements. There are many statistical methods for finding the scaling factor $\alpha$, such as the Akaike information criterion (Akaike, 1974) or the Bayesian information criterion (Schwarz, 1978). The type of the velocity that we measure, whether it is the phase velocity or the group velocity, depends on the size of the sample, size of the transducer, the direction of measurement, and the material itself (Dellinger and Vernik, 1994; Vestrum, 1994). The velocity type (phase or group) may depend on the propagation direction $n_{i}$; that dependence is determined prior to the inversion by forward modeling that is based on an initial estimate of the elastic properties. However, if the inverted stiffness tensor differs significantly from the initial estimate, an iterative approach may be required to ensure that the proper velocity type is used.

Because of the interface effect on the polarizations, computation of $\mathbf{A}$ requires one to solve the Zoeppritz equations for anisotropic media. The data that are input into these equations are the measurement direction $\mathbf{n}$, the density $\rho$ and the stiffness a of the sample, and the known stiffness and density of the medium above the interface. Given the relative complexity of the involved computations of the polarizations and velocities for each step in the minimization of the misfit function $F$, the preferred minimization algorithm is a version of random search or simulated anealing, because these algorithms do not require analytic expressions for the derivatives of $F$. To compute phase velocities $v$ corresponding to the density-scaled stiffness $\mathbf{a}$ and measurement direction $\mathbf{n}$, we use the Christoffel equations 1. To compute group (ray) velocities, we use expression 3 .

To estimate the stiffness tensor, we use our ability to separate different wave types, thereby allowing us to compute precisely the traveltime of the corresponding waves. We measured traveltimes of the three waves ( $\mathrm{P}$ - and two S-waves) along the symmetry axis and two orthogonal directions in the bedding plane, as well as along the direction [1,1,1], where the basis is given by the first three measurement directions. These traveltimes were converted to the phase velocities and used to find the stiffness tensor using the traveltime-based inversion, assuming transverse isotropy along the $z$-axis. The use of the phase velocities is well justified along the symmetry axis and the bedding plane, because along those directions the phase and group velocities are equal. The forward modeling has shown a large difference between the group and phase directions for the diagonal measurements - the group angle corresponding to the phase angle of $54.7^{\circ}$ (direction of the diagonal) is $64^{\circ}$. This shows that using measured off-angle $\mathrm{P}$-wave velocities in the phase-velocitybased inversion is not reliable. For that reason, we used the measurement of the SV velocity along the diagonal instead; for that velocity the phase and group angles are almost identical, as is shown in Figure 9. The resulting stiffness tensor in Voigt's notation is

$$
\mathbf{C}=\left(\begin{array}{cccccc}
17.6 & 9.6 & 7.7 & 0 & 0 & 0 \\
9.6 & 17.6 & 7.7 & 0 & 0 & 0 \\
7.7 & 7.7 & 12.0 & 0 & 0 & 0 \\
0 & 0 & 0 & 3.4 & 0 & 0 \\
0 & 0 & 0 & 0 & 3.4 & 0 \\
0 & 0 & 0 & 0 & 0 & 4.0
\end{array}\right) \mathrm{GPa}
$$

and the corresponding Thomsen parameters are $\gamma=0.08, \delta=0.25$, $\varepsilon=0.23$, and $\alpha=2887 \mathrm{~m} / \mathrm{s}, \beta=1548 \mathrm{~m} / \mathrm{s}$.

From this estimation of the stiffness tensor, we can find the polarizations along given directions of the phase velocities using the Christoffel equations 1 . To find the polarizations that correspond to the measured group velocity directions, we need to

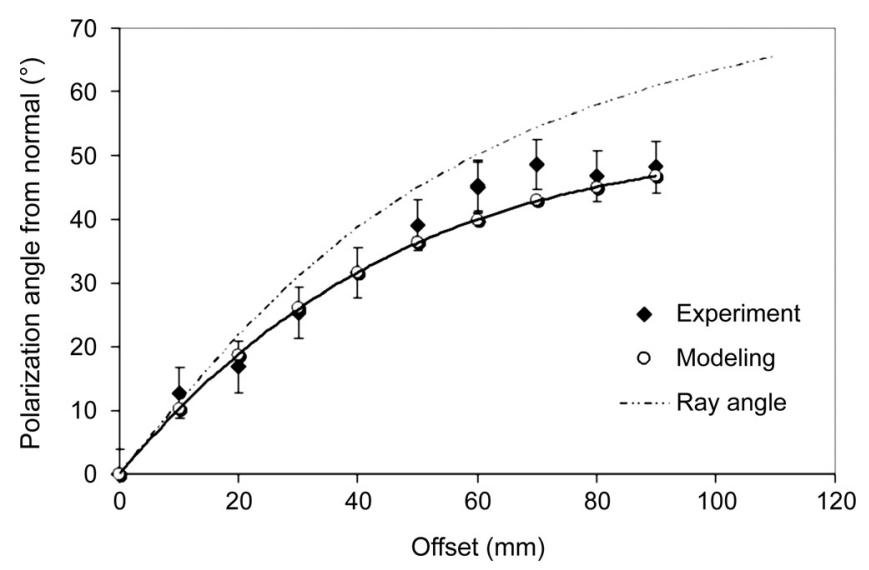

Figure 8. Polarizations of the P-wave for different offsets in the walkaway experiment (the setup of which is shown in Figure 2a). The sample thickness is $50 \mathrm{~mm}$. Uncertainty in measurement of the polarization angle (shown as bars) is estimated to be $\pm 4^{\circ}$. 


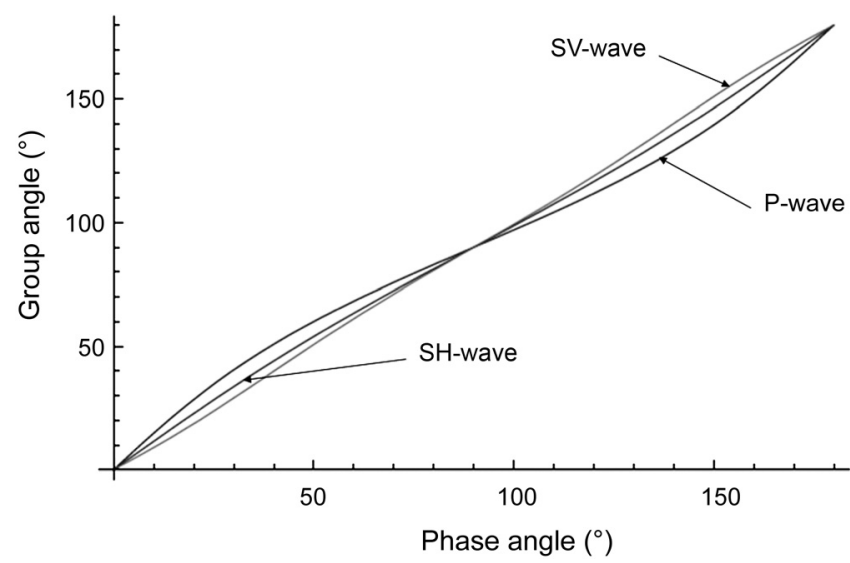

Figure 9. Calculated relation between the phase-velocity and group-velocity directions for P-, SV-, and SH-waves. The angles are measured from the symmetry axis of the paper-reinforced phenolic material used in the study.

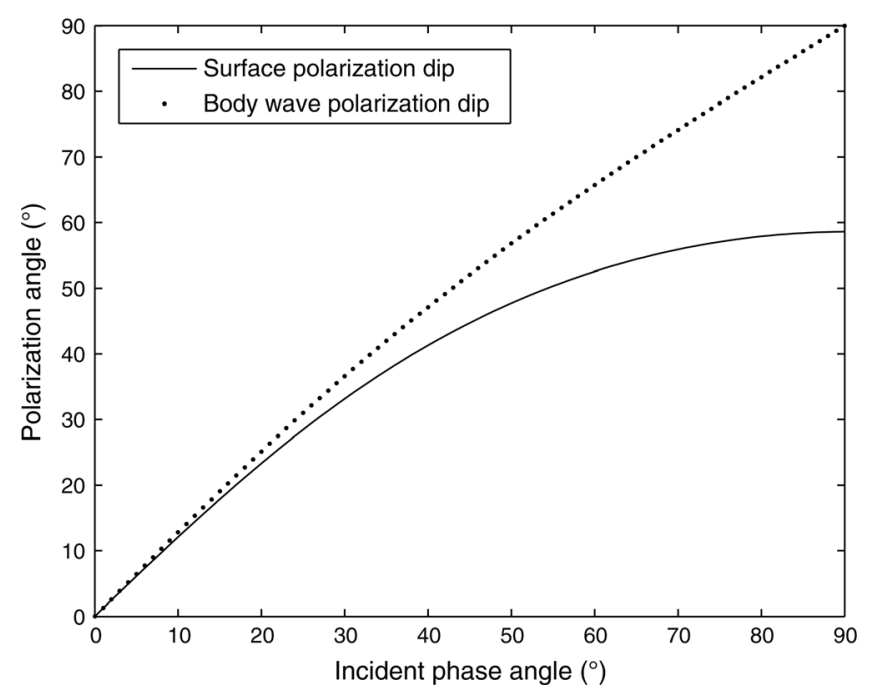

Figure 10. Graph of the polarization of the P-wave on the free surface as a function of the direction of the incident wave. The solid line represents the dip of the surface polarization, and the dotted line represents the dip of the body wave polarization. The graph was computed using the Zoeppritz equations for a free surface.

convert the group velocities to the phase velocities, which we do with the help of equation 2. With these phase velocities we can use the Zoeppritz equations to calculate the polarizations on the free surface, as shown in Figure 10. The computed polarizations as the function of the offset in the walkaway experiment are given in Figure 8, which shows good agreement between the experiment and the analytic modeling. The measured traveltimes corresponding to the group velocities of $\mathrm{P}$ - and $\mathrm{SH}$-waves are shown in Figure 11, together with the computed traveltime curves. The small discrepancy between the computed and measured traveltimes indicates that the inversion of the stiffness tensor based only on the traveltimes along the four directions is not robust enough.

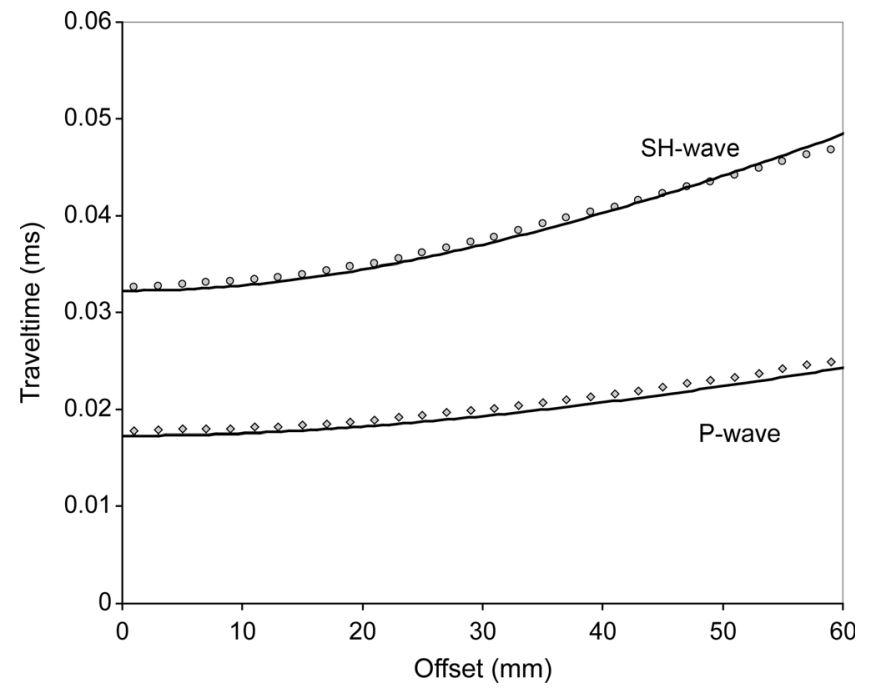

Figure 11. Traveltimes of the P- and SH-waves for the walkaway experiment, plotted against the offset. The circles indicate the measured traveltimes, whereas the lines correspond to the modeled traveltimes, which were based on the inverted stiffness tensor.

\section{CONCLUSIONS}

We have performed a walkaway laboratory experiment in which wave polarizations and group velocities were measured in a laminated material using a laser Doppler interferometer. The measured data match very well the results from analytic modeling, which used the stiffness tensor estimate from traveltimes of the three waves in different directions.

From our results, we can conclude that it is possible to characterize elasticity properties of materials from the described measurements. In particular, we are able to determine (1) the angle between the particle movement and the direction of the wave propagation, that is, the polarization, (2) the types of waves, and (3) the arrival times of the waves at the point, and (4) from those times, the wave velocities.

One of our main conclusions is that conventional estimation of TI anisotropy from laboratory measurements based on traveltimes in a limited number of directions is not very accurate. The proposed methodology for measuring the polarizations improves these inversions of the stiffness tensor.

More attention should be paid to the type of the wave source as well. Depending on the source type, the velocity is either the group velocity or the phase velocity. To eliminate any doubt about the type of the measured waves, future investigation should examine a pulse laser as a point source. Also, measurements of anisotropy under triaxial stress will be the next step of this study (Figure 1b).

Laser Doppler interferometry has an important future in laboratorymeasurements of the elasticity properties of rock samples and in physical modeling of seismic experiments.

\section{ACKNOWLEDGMENTS}

We would like to thank Patrick Rasolofosaon, two anonymous reviewers, and the associate editor for their valuable comments and suggestions that improved this paper. We also thank the sponsors 
of Curtin Reservoir Geophysics Consortium (CRGC) for their support.

\section{REFERENCES}

Akaike, H., 1974, A new look at the statistical model identification: IEEE Transactions on Automatic Control, 19, 716-723, doi:10.1109/ TAC.1974.1100705.

Aki, K., and P. Richards, 2002, Quantitative seismology: University Science Books.

Aussel, J. D., and J.-P. Monchalin, 1989, Precision laser-ultrasonic velocity measurement and elastic constant determination: Ultrasonics, 27 165-177, doi:10.1016/0041-624X(89)90059-0.

Bayón, A., and P. N. J. Rasolofosaon, 1996, Three-component recording of ultrasonic transient vibration by optical heterodyne interferometry: Journal of the Acoustical Society of America, 99, 954-961, doi:10.1121/1.414623.

Bóna, A., I. Bucataru, and M. A. Slawinski, 2008, Inversion of ray velocity and polarization for elasticity tensor: Journal of Applied Geophysics, 65, 1-5, doi:10.1016/j.jappgeo.2008.01.004.

Červený, V., 2001, Seismic ray theory: Cambridge University Press.

Dainty, J. C., 1975, Laser speckle and related phenomena: Springer Verlag.

Dellinger, J., and L. Vernik, 1994, Do traveltimes in pulse-transmission experiments yield anisotropic group or phase velocities?: Geophysics, 59, 1774-1779, doi:10.1190/1.1443564.

Dewangan, P., and V. Grechka, 2003, Inversion of multicomponent, multiazimuth, walkaway VSP data for the stiffness tensor: Geophysics, 68 1022-1031, doi:10.1190/1.1581073.

Ennos, A., 1978, Speckle interferometry, in E. Wolf, ed., Progress in optics: North-Holland, 16, 233-288.

Fukushima, Y., O. Nishizawa, H. Sato, and M. Ohtake, 2003, Laboratory study on scattering characteristics of shear waves in rock samples: Bulletin of the Seismological Society of America, 93, 253-263, doi: $10.1785 / 0120020074$.

Guilbaud, S., and B. Audoin, 1999, Measurement of the stiffness coefficients of a viscoelastic composite material with laser-generated and detected ultrasound: Journal of the Acoustical Society of America, 105, 2226-2235, doi:10.1121/1.426827.

Helbig, K., 1994, Foundations of anisotropy for exploration seismics : Handbook of geophysical exploration, I: Seismic exploration, 22: Pergamon.

Jacquot, P., and J. M. Fournier, 2000, Interferometry in speckle light: Theory and applications: Springer.

Jech, J., 1991, Computation of elastic parameters of anisotropic medium from travel times of quasi-compressional waves: Physics of the Earth and Planetary Interiors, 66, 153-159, doi:10.1016/00319201(91)90074-R.

Martin, D., B. Pouet, and P. N. J. Rasolofosaon, 1994, Laser ultrasonics applied to seismic physical modeling, in D. A. Ebrom, and J. A. McDonald, eds., Seismic physical modeling: SEG Geophysics Reprint Series $15,499-511$.

Monchalin, J.-P., 1986, Optical detection of ultrasound: IEEE Transactions on Ultrasonics, Ferroelectrics, and Frequency Control, 33, 485499, doi:10.1109/T-UFFC.1986.26860.

Monchalin, J.-P., J.-D. Aussel, R. Héon, C. K. Jen, A. Boudreault, and R. Bernier, 1989, Measurement of in-plane and out-of-plane ultrasonic displacements by optical heterodyne interferometry: Journal of Nondestructive Evaluation, 8, 121-133, doi:10.1007/BF00565636.

Nishizawa, O., T. Satoh, X. Lei, and Y. Kuwahara, 1997, Laboratory studies of seismic wave propagation in inhomogeneous media using a laser Doppler vibrometer: Bulletin of the Seismological Society of America, 87, 809-823.

Ogi, H., N. Nakamura, K. Sato, M. Hirao, and S. Uda, 2003, Elastic, anelastic, and piezoelectric coefficients of langasite: Resonance ultrasound spectroscopy with laser-Doppler interferometry: IEEE Transactions on Ultrasonics, Ferroelectrics and Frequency Control, 50, 553-560.

Pouet, B., and P. N. J. Rasolofosaon, 1990, Seismic physical modeling using laser ultrasonics: 60th Annual International Meeting, SEG, Expanded Abstracts, 841-844.

Pouet, B., and P. N. J. Rasolofosaon, 1993, Measurement of broadband intrinsic ultrasonic attenuation and dispersion in solids with laser techniques: Journal of the Acoustical Society of America, 93, 1286-1292 doi:10.1121/1.405413.

Pros, Z., and V. Babuska, 1967, A method for investigating the elastic anisotropy on spherical rock samples: Zeitschrift für Geophysik, 33, 289291.

Rasolofosaon, P. N. J., D. Martin, F. Gascon, A. Bayón, and A. Varade, 1994, Physical modeling of 3D seismic wave propagation, in K. Helbig, ed., Modeling the earth for oil exploration: Final report of the CEC's Geoscience I Program, 1990-93: Pergamon Press, 637-686.

Rasolofosaon, P. N. J., and B. E. Zinszner, 2002, Comparison between permeability anisotropy and elasticity anisotropy of reservoir rocks: Geophysics, 67, 230-240, doi:10.1190/1.1451647.

Schwarz, G. E., 1978, Estimating the dimension of a model: Annals of Statistics, 6, 461-464, doi:10.1214/aos/1176344136.

Scruby, C. B., and L. E. Drain, 1990, Laser ultrasonics - Techniques and application: Adam Hilger.

Siggins, A. F., and D. N. Dewhurst, 2007, Ultrasonic determination of the elastic constants of VTI shale: Proceedings of the International Congress on Ultrasonics, 2007, Paper ID 1075, doi:10.3728/ICUltrasonics. 2007.

Vestrum, R. W., 1994, Group and phase-velocity inversions for the general anisotropic stiffness tensor: M.S. thesis, University of Calgary. 